\title{
Modeling of Electric Disturbance Signals Using Damped Sinusoids via Atomic Decompositions and Its Applications
}

\author{
Lisandro Lovisolo, ${ }^{1}$ Michel P. Tcheou, ${ }^{2,}{ }^{3}$ Eduardo A. B. da Silva, ${ }^{2}$ \\ Marco A. M. Rodrigues, ${ }^{3}$ and Paulo S. R. Diniz ${ }^{2}$ \\ ${ }^{1}$ Departamento de Eletrônica e Telecomunicações (DETEL), Faculdade de Engenharia (FEN), \\ Universidade do Estado do Rio de Janeiro (UERJ), Rio de Janeiro 20550-900, RJ, Brazil \\ ${ }^{2}$ Laboratory of Signal Processing, PEE/COPPE and DEL/Poli, Federal University of Rio de Janeiro, CP 68504, \\ Rio de Janeiro 21941-972, RJ, Brazil \\ ${ }^{3}$ Electric Power Research Center (CEPEL), CP 68007, Rio de Janeiro 21941-590, RJ, Brazil
}

Received 10 August 2006; Accepted 17 December 2006

Recommended by Alexander Mamishev

The number of waveforms monitored in power systems is increasing rapidly. This creates a demand for computational tools that aid in the analysis of the phenomena and also that allow efficient transmission and storage of the information acquired. In this context, signal processing techniques play a fundamental role. This work is a tutorial reviewing the principles and applications of atomic signal modeling of electric disturbance signals. The disturbance signal is modeled using a linear combination of damped sinusoidal components which are closely related to the phenomena typically observed in power systems. The signal model obtained is then employed for disturbance signal denoising, filtering of "DC components," and compression.

Copyright ( $\odot 2007$ Lisandro Lovisolo et al. This is an open access article distributed under the Creative Commons Attribution License, which permits unrestricted use, distribution, and reproduction in any medium, provided the original work is properly cited.

\section{INTRODUCTION}

Electric disturbance signals are acquired by digitizing the voltage and/or current waveforms with digital fault recorders (DFRs) at several points of the power system network. Figure 1 illustrates a typical DFR data, composed by the voltage and current waveforms of a three-phase system and the correspondent neutrals in a transmission line. In Figure 1, we can observe the three main parts of interest for fault analysis. The prefault shows the system behavior prior to the fault occurrence and the postfault shows the system state after fault recovering. Along with fault signals, power quality events are also acquired in order to monitor transient behavior and evaluate the impacts of power consumer apparatuses on the power quality. The analysis of disturbance signals allows the identification of patterns and characteristics of faults and also to assess power quality [1-6].

The number of points monitored in power systems is increasing rapidly because: (a) the power system operation bounds get more critical as demand increases; (b) at large interconnected systems, it is necessary to establish precisely the causes of the disturbance as well as the responsibilities for the resulting effects. Storage and transmission of disturbance signals may generate an information overload, even though the cost of storage is decreasing rapidly, the general tendency is to sample signals at higher rates and for longer periods of time. Thus, storage capacity and transmission bandwidth problems persist, demanding good compression schemes. Also, the information overload is a serious problem to disturbance analysis, as human experts (that perform the analysis) have in general difficulty to analyze very large amounts of data. This creates a demand for computational tools (i) that aid in the analysis of the phenomena; (ii) that allow efficient transmission and storage of the information. Very different signal processing techniques have been applied to analyze and compress disturbance signals [5, 7-22]. The results of the application of signal processing techniques in this analysis are so rich and fruitful that specific hardware for these tasks is being developed [23].

This work is a tutorial reviewing the principles and applications of atomic signal modeling of electric disturbance signals which was first presented in [22]. This atomic decomposition decomposes/models a signal using a linear combination of damped sinusoidal components which are closely 


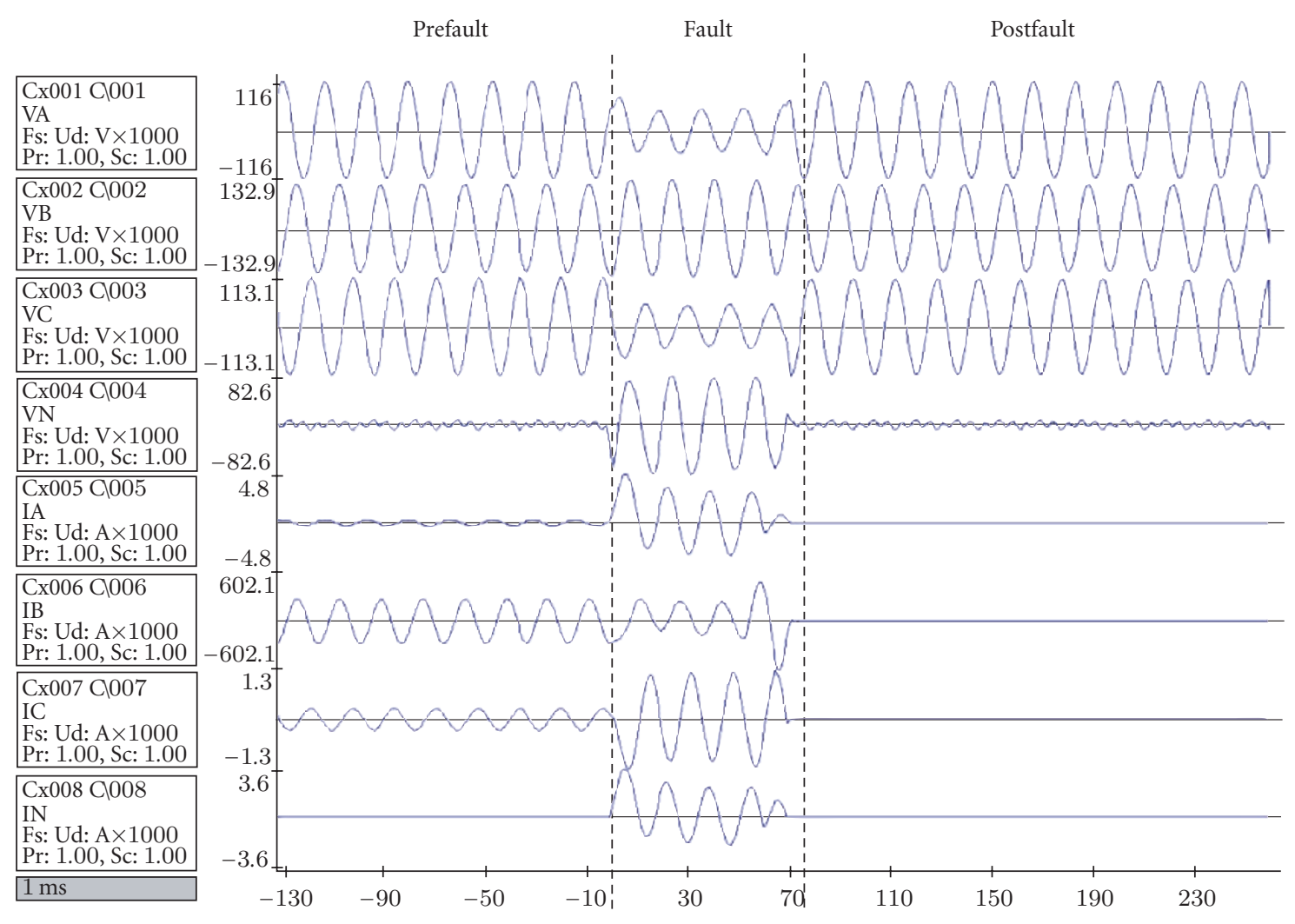

Figure 1: Typical data acquired by a digital fault recorder.

related to the phenomena typically observed at power systems. That is, the components employed are coherent to power system phenomena. The signal components, each one associated to a different phenomenon, are identified through an atomic decomposition algorithm. The algorithm employed is based on the matching pursuit (MP) [24-28]. This strategy identifies the different natural phenomena represented in the signal that originated during the disturbance.

\section{Paper organization}

The damped sinusoids signal model is discussed in Section 2. Atomic decompositions are discussed in Section 3. The decomposition algorithm is described in Section 4 along with some examples and a brief discussion of the improvements implemented with respect to the work in [22]. In Section 5, we discuss some applications of the atomic decompositions obtained using this algorithm. These applications include coherent signal modeling, signal denoising, nonlinear filtering of the so-called "DC component," and a compression scheme for disturbance signals. Section 6 closes the paper.

\section{DAMPED SINUSOIDAL MODELING OF DISTURBANCE SIGNALS}

Regardless of the quantities measured, the aim of power system monitoring is to study the evolution in time of disturbance phenomena. These phenomena are represented, in general, as sinusoidal oscillations of increasing or decreasing amplitudes, and are highly influenced by circuit switching, as well as by nonlinear equipments. In order to analyze and compress signals from power systems, it is important to use a model that is capable of precisely representing the components that may compose those signals. Xu [29] discusses common phenomena in power systems.

(i) Harmonics are low-frequency phenomena ranging from the system fundamental frequency $(50 / 60 \mathrm{~Hz})$ to $3000 \mathrm{~Hz}$. Their main sources are semiconductor apparatuses (power electronic devices), arc furnaces, transformers (due to their nonlinear flux-current characteristics), rotational machines, and aggregate loads (a group of loads treated as a single component).

(ii) Transients are observed as impulses or high-frequency oscillations superimposed to the voltages or currents of fundamental frequency $(50 / 60 \mathrm{~Hz})$ and also exponential DC and modulated components. The more common sources of transients are lightnings, transmission line, and equipment faults, as well as switching operations, although transients are not restricted to these sources. Their frequency range may span up to hundreds of thousands of $\mathrm{Hz}$, although the measurement system (and the power line) usually filters components above few thousands of $\mathrm{Hz}$.

(iii) Swells and Sags are increments or decrements, respectively, in the RMS voltage of duration from half cycle to 1 minute (approximately). 


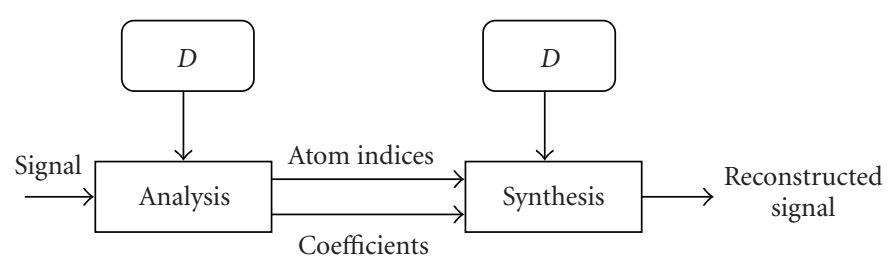

FIgURE 2: Signal analysis and synthesis based on atomic signal decompositions using a dictionary $D$.

When analyzing disturbance signals, it is interesting to be capable of detecting, modeling, and identifying those phenomena. Some techniques commonly employed for modeling and analyzing power disturbance signals are Fourier filtering $[9,10]$; Prony analysis $[11,12]$; autoregressive moving average models [7]; state-space tracking methods [7]; wavelets [11, 13-19]. In some cases, these methods are used along with artificial intelligence strategies $[8,12,30,31]$.

Roughly, one can consider that electric power systems are basically formed by sources, loads, and transmission lines, that is, RLC circuits, whose transient behaviors are modeled by damped sinusoids. In addition, discontinuities may appear in these signals due to circuit switching. Following these premises, a disturbance signal $x(t)$ can be approximated by $[22,32,33]$

$$
\begin{aligned}
x(t)= & \sum_{m=1}^{M} \alpha_{m} e^{-\rho_{m}\left(t-t_{m}^{s}\right)} \cos \left(2 \pi k_{m} F t+\phi_{m}\right) \\
& \times\left[u\left(t-t_{m}^{s}\right)-u\left(t-t_{m}^{e}\right)\right],
\end{aligned}
$$

where $M$ is the number of expansion elements, $F$ is the fundamental frequency $(50 / 60 \mathrm{~Hz}), u(\cdot)$ corresponds to the unit step function, and each element is represented by a 6-tuple $\left(\alpha_{m}, k_{m}, \rho_{m}, \phi_{m}, t_{m}^{s}, t_{m}^{e}\right)$. In this 6-tuple, $\alpha_{m}$ is the amplitude, $k_{m}$ is an integer multiple of the fundamental frequency, $\rho_{m}$ is the decaying factor, $\phi_{m}$ is the phase, and $t_{m}^{s}$ and $t_{m}^{e}$ are, respectively, the starting and ending times of the $m$ th signal component.

The well-known Prony method $[7,11,12]$ largely employed for analyzing power system signals obtains a similar model. However, the Prony method does not consider that distinct damped sinusoids can start at different time instants neither that they can have different time supports. Therefore, the proposed model adds a time localization feature to Prony analysis.

In the signal processing community, damped sinusoids are present in several applications. For example, in [3436] such components were used for transient detection and analysis. The large amount of potential applications of such components is motivated by the fact that damped sinusoids are solutions for ordinary differential equations that often appear in physical system models [27, 28, 37, 38]. For a long time, researchers have been designing systems and algorithms to estimate the parameters of damped sinusoids embedded in several signals [39-46].

In $[21,47]$, disturbance signals are modeled using a fundamental, a set of harmonics, and after subtracting these components from the signal, the resulting signal is decom- posed using a wavelet transform. The signal model in (1) differs from those in $[21,47]$, since it does not restrict the fundamental and the harmonics to have constant amplitudes neither full nor the same time support.

How can one represent a given signal in accordance to the signal model in (1)? For that purpose, we employ an adaptive atomic decomposition algorithm. Before discussing the algorithm, we address some important concepts of atomic decompositions.

\section{ATOMIC DECOMPOSITIONS}

Define a dictionary $D$ as the set of all possible structures, predefined waveforms, that can be used to represent signals. The aim of atomic signal decomposition algorithms is to select a subset of $M$ elements $\mathbf{g}_{\gamma(m)}$ from the dictionary that approximates $\mathbf{x}$ using the linear combination given by the $M$-term approximation or representation (or simply, $M$-term)

$$
\mathbf{x} \approx \hat{\mathbf{x}}=\sum_{m=1}^{M} \alpha_{m} \mathbf{g}_{\gamma(m)}, \quad \mathbf{g}_{\gamma(m)} \in D
$$

The atoms $\mathbf{g}_{\gamma(m)}$ in the $M$-term are indexed by the mapping $\gamma(m)$ that is defined as $\gamma: \mathbb{Z}^{+} \rightarrow\left\{1, \ldots, \#_{D}\right\} ; \#_{D}$ is the dictionary cardinality - the number of elements in $D$, thus $\gamma(m) \in\left\{1, \ldots, \#_{D}\right\}$. The parameter $\alpha_{m}$ denotes the coefficient, that is, the weight of $\mathbf{g}_{\gamma(m)}$, and $M$ is the number of atoms used to approximate $\mathbf{x}$. The $M$-term representation of a signal is the result of an analysis-synthesis procedure which is illustrated in Figure 2. The analysis of the signal obtains the coefficients and atom indices while the synthesis of the signal is accomplished using (2).

Atomic representations differ from classical transformbased signal representations, because the atoms used in the $M$-term may be linearly dependent. In addition, since, in general, $D$ has more elements than necessary to span the signal space, the selection of the atoms may be signaldependent, leading to an adaptive signal decomposition (analysis-synthesis).

Atomic representations have been employed for signal filtering and denoising $[25,48]$, analysis of the physical phenomena behind the observed signal together with pattern recognition and signal modeling [25, 27, 28, 49-52], timefrequency analysis $[24,25]$, and harmonic analysis $[52,53]$. Atomic representations can also provide good signal compression tools [53-57]. Recently, atomic representations were used to discriminate outcomes from different Gaussian processes [58]. 
The distortion of the $M$-term approximation of a signal $\mathbf{x}$ is

$$
d(\mathbf{x}, M, D)=\|\mathbf{x}-\hat{\mathbf{x}}\|=\left\|\mathbf{x}-\sum_{m=1}^{M} \alpha_{m} \mathbf{g}_{\gamma(m)}\right\| .
$$

This distortion depends on (i) the number of elements $M$ used to represent $\mathbf{x}$; (ii) the atoms $\mathbf{g}_{\gamma(m)}$ used to express the signal; (iii) and the weights $\alpha_{m}$ of the atoms. Since $D$ defines the atoms that can be used in the $M$-term, the distortion depends on $D$. For $M$-terms that use atoms from a dictionary $D$ being capable of representing any signal $\mathbf{x} \in \mathbb{X}$ with an arbitrarily distortion $d(\mathbf{x}, M, D), D$ must be complete in $\mathbb{X}$ $[25,59-61]$. That is, there will be at least one linear combination of elements from $D$ that gives $\widehat{\mathbf{x}}=\mathbf{x}$, for all $\mathbf{x} \in \mathbb{X}$, that is, $D$ must span $\mathbb{X}$. When $D$ has more elements than necessary to span the signal space, it is said to be overcomplete or redundant $[25,54,59,61]$.

Ideally, the atoms used in the $M$-term expansion should depend on the signal, and in this case the decomposition is said to be adaptive $[24,25,27,51,59,60,62]$. Since an overcomplete dictionary allows expressing the same signal using different $M$-terms (the representation is not unique), an overcomplete or redundant dictionary is a requirement if adaptive signal decompositions are desired. Ideally, adaptive approximations should discriminate the relevant information represented in the signal ignoring noise, being the relevant information defined by the dictionary atoms.

Most signal processing applications deal with outcomes from physical processes. In these cases, the observed signal $\mathbf{x}$ is a mixture of components $\mathbf{p}_{m}$, representing physical phenomena, given by

$$
\mathbf{x}=\sum_{m} \beta_{m} \mathbf{p}_{m}+\mathbf{n}
$$

where $\mathbf{n}$ is the noise, inherent to the measurement process. From the perspective of signal modeling, it is interesting for the atoms $\mathbf{g}_{\gamma(m)}$ used to approximate the signal to be similar to the phenomena $\mathbf{p}_{m}$ that are represented in $\mathbf{x}$. The closer the selected dictionary elements $\mathbf{g}_{\gamma(m)}$ and weights $\alpha_{m}$ are to the physical phenomena $\mathbf{p}_{m}$ and weights $\beta_{m}$, the better is the signal expansion for modeling and pattern recognition purposes. We say that the representation is coherent to the signal when it is a meaningful signal model.

The most compact or sparse representation of $\mathbf{x}$ is the one using the smallest number of atoms $[25,61]$ with null distortion. However, in practice, a small number of terms $M$ providing an acceptable distortion may suffice for representing the signal in a sparse manner.

In essence, atomic decompositions may provide an accurate, sparse, and coherent signal model with low distortion. A very popular algorithm to obtain atomic decompositions is the matching pursuit (MP) $[24,25]$.

\subsection{Matching pursuit}

The MP $[24,25]$ approximates signals iteratively finding the best possible approximation at each iteration. The MP has emerged more or less at the same time in several scientific fields, for example, in signal processing in [63], in statistics in $[64,65]$, and in control applications [66].

Let $D=\left\{\mathbf{g}_{\gamma}\right\}$ and $\gamma \in\left\{1, \ldots, \#_{D}\right\}$ such that $\left\|\mathbf{g}_{\gamma}\right\|=1$ for all $k$, and let $\#_{D}$ be dictionary cardinality, that is, the number of elements in $D$. In each decomposition step or iteration $m \geq 1$, the MP searches for the atom $\mathbf{g}_{\gamma(m)} \in D$, that is, $\gamma(m) \in\left\{1, \ldots, \#_{D}\right\}$, with largest inner product with the residual signal $\mathbf{r}_{\mathbf{x}}^{m-1}[24,25]$. The initial residue is set to be $\mathbf{r}_{\mathbf{x}}^{0}=\mathbf{x}$. The selected atom $\mathbf{g}_{\gamma(m)}$ is then subtracted from the residue to obtain a new residue

$$
\mathbf{r}_{\mathbf{x}}^{m}=\mathbf{r}_{\mathbf{x}}^{m-1}-\alpha_{m} \mathbf{g}_{\gamma(m)}, \quad \alpha_{m}=\left\langle\mathbf{r}_{\mathbf{x}}^{m-1}, \mathbf{g}_{\gamma(m)}\right\rangle .
$$

The MP obtains the $M$-term signal representation/approximation of (2) with distortion $\mathbf{r}_{\mathbf{x}}^{M}=\mathbf{x}-\hat{\mathbf{x}}$ (the $M$ th residue). In practice, the decomposition step (the calculation of $\alpha_{m}$, $\gamma(m)$, and the residue $\left.\mathbf{r}_{\mathbf{x}}^{m}\right)$ is iterated until a prescribed distortion $\left(\left\|\mathbf{r}_{\mathbf{x}}^{m}\right\|\right)$, a maximum number of steps $M$, or a minimum for an approximation metric are reached $[22,24,25,60]$.

\section{Local fitting}

Due to its greediness $[67,68]$, the MP algorithm confuses signal components [69]. This happens because the MP searches for the atom that best matches the overall signal, which may produce a bad local fitting. For example, to solve this drawback, the high-resolution pursuits (HRP) $[51,70]$ use Bspline windows to locally fit the atom found by the MP to the residue. The algorithm in Section 4 uses a local fitting strategy for eliminating pre-echo and post-echo artifacts that often appear in MP-like algorithms, which is accomplished by windowing the atoms with a rectangular window. In addition, this algorithm includes a set of heuristics inside the MP loop to instruct the MP for correct atom selection.

The MP is capable of obtaining compact and efficient signal representations. However, an important aspect for that is the dictionary, since the elements in it should be coherent to the components represented in the signal.

\subsection{Parameterized dictionaries}

If the class of components that may be represented in the signal is previously known, then it would be wise to use a dictionary containing atoms that resemble these components $[25,59,71]$. A common strategy is to define the dictionary elements from a set of prototype functions/signals. In such dictionaries, the actual waveforms of the dictionary atoms depend on a set of parameters modifying the prototype signal. These dictionaries are said to be parameterized since each dictionary element $\mathbf{g}_{\gamma}$ is defined by a given value of the parameter set $\Gamma$, that is,

$$
\gamma \in \Gamma=\left\{\gamma_{0}, \gamma_{1}, \ldots, \gamma_{\#_{D}-1}\right\},
$$

where $\#_{D}$ is the number of possible distinct parameter set values defining different atoms $\mathbf{g}_{\gamma}$ and $\Gamma$ is the set of all possible parameters. For example, the popular Gabor dictionary [24$27,51,60,72-74]$ is composed by Gaussian shaped atoms in 


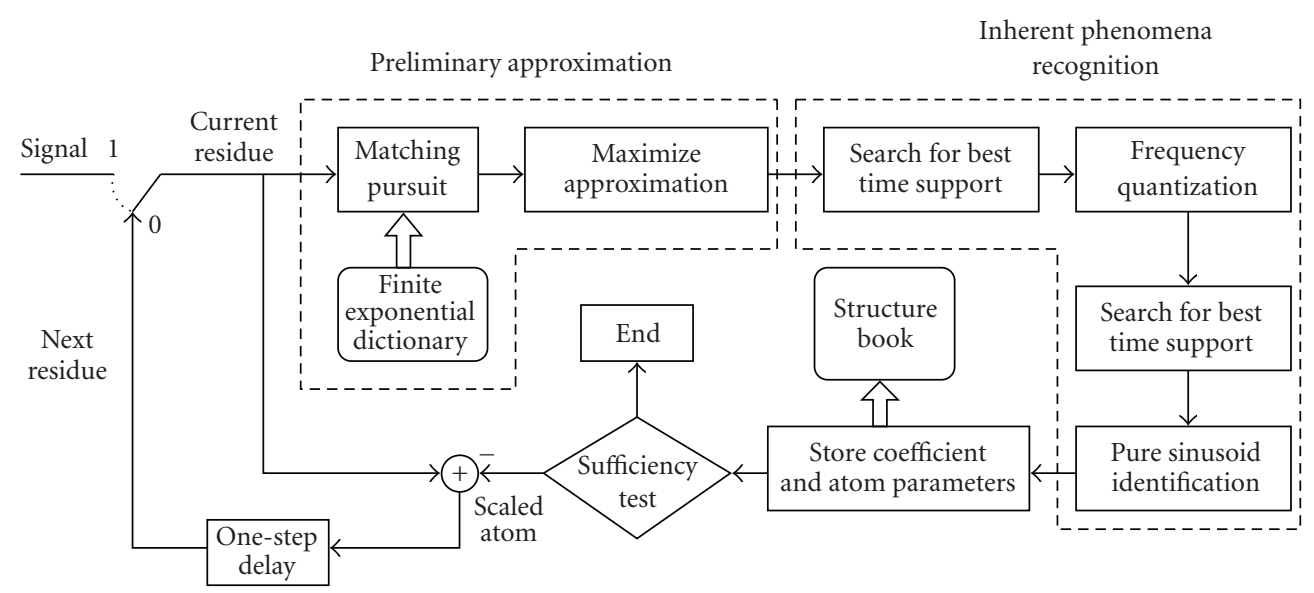

FIGURE 3: Block diagram of the atomic decomposition algorithm. In the first iteration, the switch is in position 1 and in the remaining iterations, it stays in position 0 .

different scales, with varying centers in time and, multiplied by different complex sinusoids, the so-called time-frequency atoms $[24,25,75]$.

The use of a parameterized dictionary allows for estimating the signal and obtaining coherent decompositions. For example, parameterized dictionaries were employed for pattern recognition [51] and signal modeling [49, 76] using atomic decompositions. The decomposition algorithm in Section 4 employs a parameterized dictionary of damped sinusoids in order to obtain an atomic signal model according to (1).

\section{Continuous parameters}

In some cases, one may have to adapt or fit the structures used in the signal representation to the actual signal being decomposed. For that purpose, the parameter set value $\gamma$ defining an atom could be any point inside a region of the parameter space instead of one chosen from a set of $\#_{D}$ values. In this case, it is said that the parameters of the atoms are continuous. In general, to obtain continuous parameter atoms, one uses optimization algorithms to find the values of the parameter set defining each atom in the $M$-term. One starts the optimization using a guess for the atom parameters, which is obtained from a finite cardinality dictionary. The decomposition algorithm in Section 4 employs this approach.

\section{DECOMPOSITION ALGORITHM}

This section presents an atomic decomposition algorithm that obtains the signal representations in accordance with the signal model in (1). The algorithm is based on the MP and uses a parameterized dictionary of damped sinusoids with continuous parameters. The simple use of the MP with a parameterized dictionary of damped sinusoids does not grant obtaining a good signal model. To improve the signal modeling, a set of heuristics is introduced in the decomposition loop in order to guide the atom selection. The procedure described here derives from the one in [22].

The elements of the parameterized damped sinusoidal atom $\mathbf{g}_{\gamma}$ are given by

$$
\begin{gathered}
g_{\gamma}(n)=K_{\gamma} g(n) \cos (\xi n+\phi)\left[u\left(n-n^{s}\right)-u\left(n-n^{e}\right)\right], \\
n=\{0, \ldots, N-1\}, \\
g(n)=\left\{\begin{array}{lc}
1 & \text { if } \rho=0 \text { pure sinusoid }(\xi \neq 0), \\
e^{-\rho\left(n-n^{s}\right)} & \text { if } \rho>0 \text { decreasing exponential, } \\
e^{\rho\left(n^{e}-n\right)} & \text { if } \rho<0 \text { increasing exponential, }
\end{array}\right.
\end{gathered}
$$

where $K_{\gamma}$ is set so that $\left\|\mathbf{g}_{\gamma}\right\|=1$ and $N$ is the signal length. Furthermore, the atom in (7) is defined by the 5-tuple $\gamma=$ $\left(\rho, \xi, \phi, n^{s}, n^{e}\right)$ in which $\rho$ is the decaying factor, $\xi$ denotes the frequency, $\phi$ denotes the phase, $n^{s}$ and $n^{e}$ are the starting and ending samples. The phase of the atom is optimized to provide the maximum inner product between the atom and the residue in every iteration $[22,26]$.

Figure 3 shows the block diagram of the decomposition algorithm. First, the algorithm searches the atom having the largest correlation with the residue in a finite exponential dictionary with presampled parameter space. The elements of this dictionary are given by

$$
\begin{aligned}
& g_{\gamma_{d}}(n)=g_{j}\left(n-2 p^{j}\right) \cos \left(n k \pi 2^{1-j}+\phi\right), \quad n=\{0, \ldots, N-1\}, \\
& g_{j}(n)= \begin{cases}\delta(j), & j=0, \\
K_{\gamma_{d}} e^{ \pm n 2^{-j}}, & j \in[1, L), \\
\frac{1}{\sqrt{N}}, & j=L .\end{cases}
\end{aligned}
$$

This dictionary has $L=\log _{2}(N)$ scales and the ranges of the parameters that define the elements are $j \in[0, L], p \in$ $\left[0, N 2^{-j}\right)$, and $k \in\left[0,2^{j}\right)$, while the phase $\phi$ is optimized. 


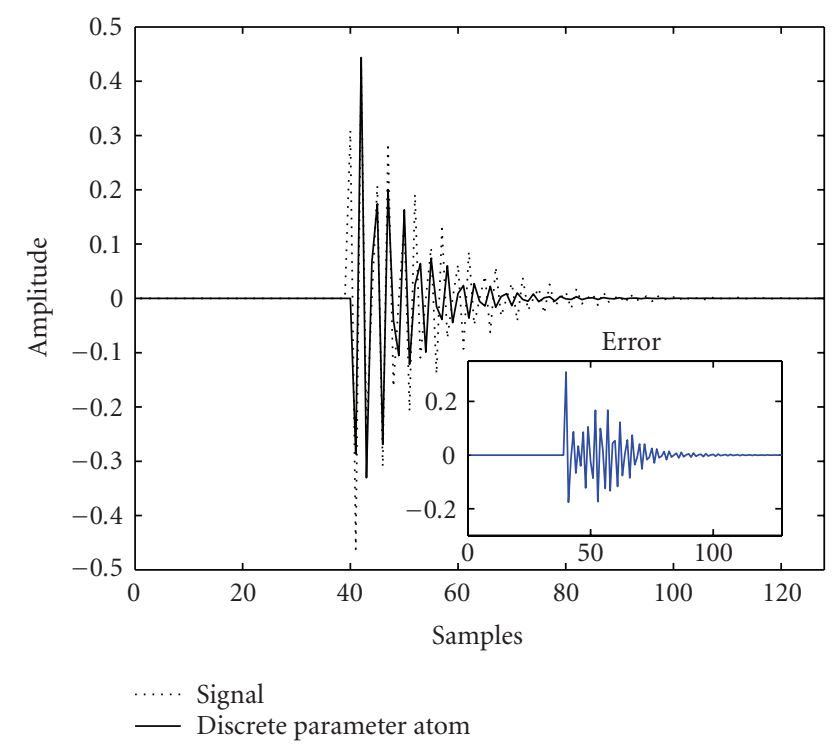

(a) Atom found using the discrete parameter dictionary

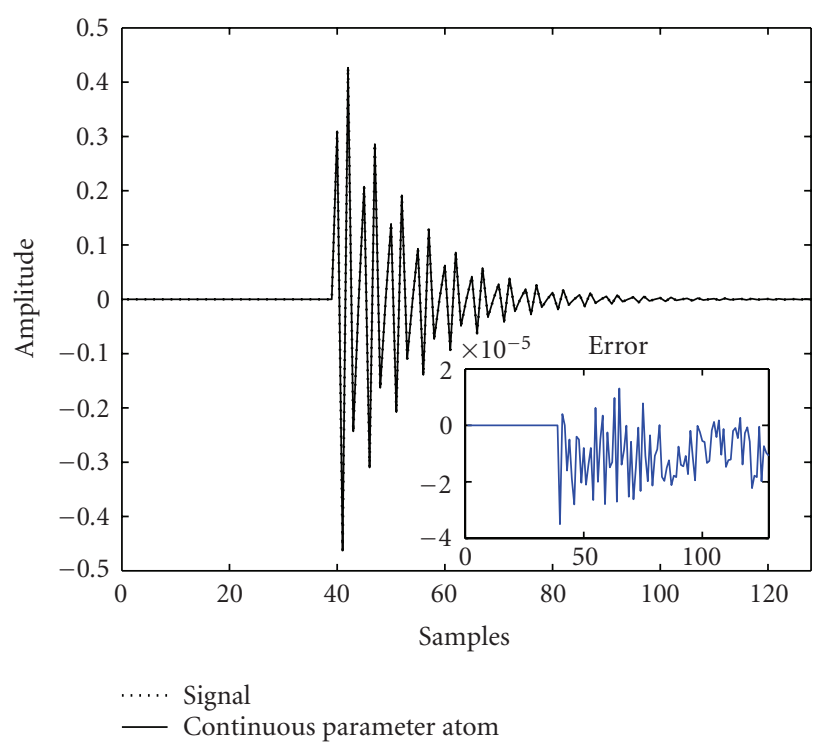

(b) Atom found after optimization of the atom parameters

FIGURE 4: Result of the optimization of the atoms parameters.

The discrete parameters found for the atom $\gamma_{d}$ are then optimized to find the $\gamma$ from a set of continuous parameters maximizing the match between the atom and the current residue using a Newton-like optimization method [22]. Figure 4 illustrates the result of this optimization.

The simple use of the MP with a damped sinusoid dictionary does not guarantee the generation of a coherent decomposition (a physically interpretable representation with respect to the phenomena in disturbance signals). Figure 5 shows an example of what occurs when a fault signal is decomposed by the MP using a damped sinusoid dictionary. The fault occurs after the 200th sample of the signal. However, the atom found does not represent the fault.

Aiming at a coherent decomposition, after selecting a damped sinusoid to approximate the atom, the algorithm performs inherent phenomena recognition by reducing the time support of the atom (determined by $n^{s}$ and $n^{e}$ ). The region of support of the atom is reduced sample by sample by box-windowing the atom in order to verify whether a new time support produces better fit between the atom and the current residue.

The next step of the decomposition algorithm is to quantize the atom frequency to a multiple of the fundamental and repeat the time support search for the new quantized frequency. After that, the algorithm decides if it is worth to use a pure sinusoid instead of a damped one. This decision relies on a heuristic that is based on a similarity metric. The heuristic (decision criterion) is basically a tradeoff between the error per sample of the resulting residue in the region of support of the atom and the inner product of the atom with the current residue [22].

Figure 6 shows how the whole decomposition algorithm behaves in the first four decomposition steps for a natural disturbance signal from [77]. The first residue is the signal

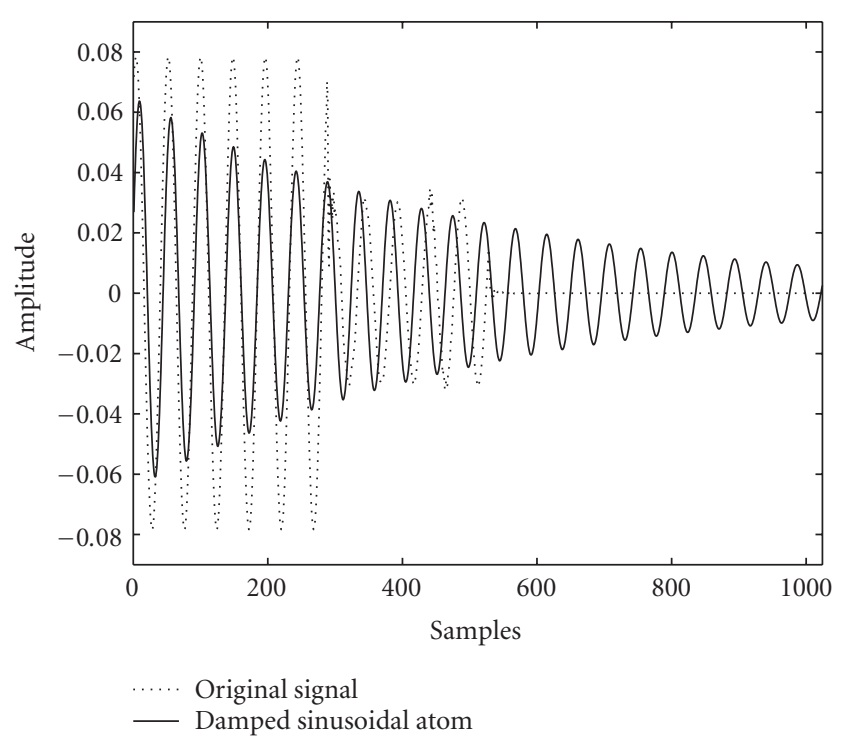

FIgURE 5: Failure of the MP in finding coherent structures.

itself. Note that the components found in each iteration of the algorithm closely match the correspondent residues.

The decomposition algorithm stops when the approximation achieved is good enough. Otherwise, it scales and subtracts the atom from the current residue and produces a new residue to be approximated in the following iteration. To decide if the decomposition should or should not continue, we employ the following criterion: is there any dictionary atom sufficiently coherent to the remaining residue? If the answer is yes, the decomposition continues; otherwise it stops. To answer this question, we measure if the dictionary 


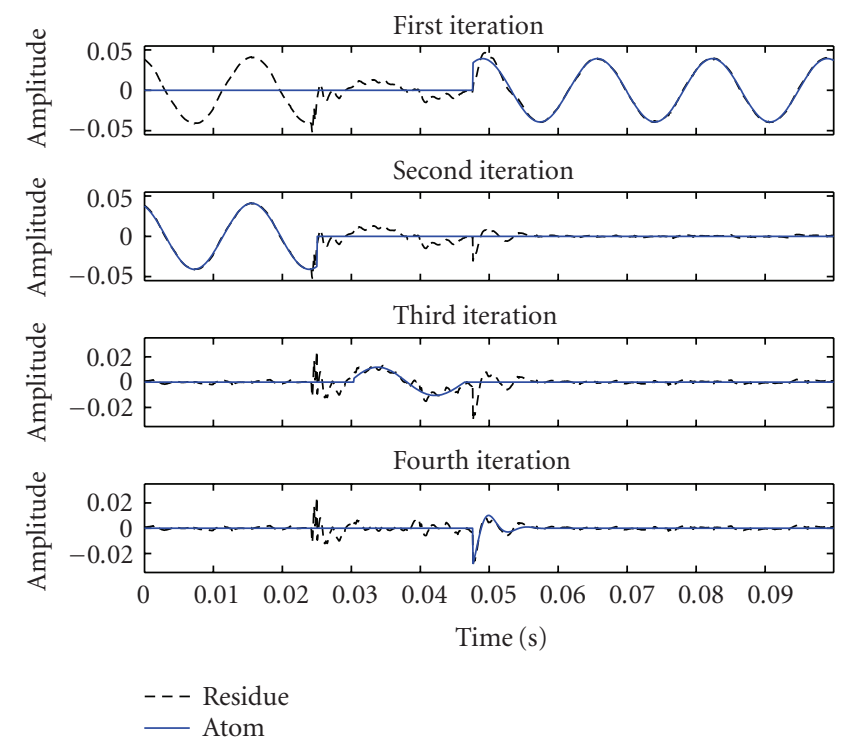

Figure 6: Finding of the coherent structures for a natural disturbance signal available in [77].

atoms are capable of providing a good signal approximation. For that, we employ the approximation ratio $[24,60]$

$$
\lambda(m)=\frac{\left|\left\langle\mathbf{r}_{\mathbf{x}}^{m-1}, \mathbf{g}_{\gamma(m)}\right\rangle\right|}{\left\|\mathbf{r}_{\mathbf{x}}^{m-1}\right\|} .
$$

It measures how much of the residue $\mathbf{r}_{\mathbf{x}}^{m-1}$ is approximated at the step $m$. Note that the residue norm $\left\|\mathbf{r}_{\mathbf{x}}^{m-1}\right\|$ is a measure of the approximation error, but it does not measure if the residue is still highly correlated to any atom in the dictionary. The coefficient magnitude $\left|\left\langle\mathbf{r}_{\mathbf{x}}^{m-1}, \mathbf{g}_{\gamma(m)}\right\rangle\right|$ depends on the residue energy since the atoms have unit norm. Therefore, if one employs $\left|\left\langle\mathbf{r}_{\mathbf{x}}^{m-1}, \mathbf{g}_{\gamma(m)}\right\rangle\right|$ as halting criterion, the halting would also be influenced by the residue norm. The use of the approximation ratio eliminates such influence [22].

At the end of the decomposition algorithm, we obtain the signal approximation in (1) represented by the sequence of pairs $\left(\alpha_{m}, \gamma(m)\right), m=0, \ldots, M-1$, where $\gamma(m)=$ $\left(\rho_{m}, \xi_{m}, \phi_{m}, n_{m}^{s}, n_{m}^{e}\right)$ (see (1) and (7)). Note that the algorithm delivers discrete values for the atom parameters $n_{m}^{s}, n_{m}^{e}$, and $\xi_{m}$, while the remaining parameters of the atom $\rho_{m}$ and $\phi_{m}$ and the atom amplitude $\alpha_{m}$ are continuous.

\section{APPLICATIONS OF THE SIGNAL MODEL AND THE DECOMPOSITION ALGORITHM}

\subsection{Coherent signal modeling}

What happens if the signal to be decomposed is acquired in a severe noise environment? Ideally, one wants the signal components to be identified in spite of the noise that may be added to the signal. However, if the noise has an energy that is comparable to the energy of a given component, then it would be difficult to distinguish between them. We address now how the decomposition algorithm presented performs in detecting the signal components when the signal is corrupted by noise.

Define the noisy signal

$$
\mathbf{x}_{\text {noise }}=\mathbf{x}+\mathbf{n} \text {, }
$$

where $\mathbf{n}$ is any noise signal. From this definition, we can compute

$$
\mathrm{SNR}_{\mathrm{C}}=10 \log _{10}\left(\frac{\|\mathbf{x}\|^{2}}{\|\mathbf{n}\|^{2}}\right)=10 \log _{10}\left(\frac{\|\mathbf{x}\|^{2}}{\left\|\mathbf{x}-\mathbf{x}_{\text {noise }}\right\|^{2}}\right)(\mathrm{dB})
$$

to measure how much $\mathbf{x}$ is corrupted by noise. Figure 7 shows the components identified in a given signal corrupted by noise signals with different levels of $\mathrm{SNR}_{\mathrm{C}}$ by the decomposition algorithm of the previous section. The original synthetic signal (uncorrupted by noise) is shown at the top of Figure $7(\mathrm{a})$ and the components used in its generation are at the bottom of Figure 7(a). Figure 7(b) shows the signal in Figure 7 (a) corrupted with noise such that $\mathrm{SNR}_{\mathrm{C}}=30 \mathrm{~dB}$ and the structures found by the decomposition algorithm. Note that they are very similar to the ones used to generate the signal. Figures $7(\mathrm{c})$ and $7(\mathrm{~d})$ show the same signal corrupted by noise such that $\mathrm{SNR}_{\mathrm{C}}=20 \mathrm{~dB}$ and $\mathrm{SNR}_{\mathrm{C}}=10 \mathrm{~dB}$, respectively. One notes that in these cases, the three structures of larger energy are identified, but the fourth is not. The energy of the fourth structure is indeed smaller than the one of the noise in these cases. When the noise added to the signal is such that $\mathrm{SNR}_{\mathrm{C}}=5 \mathrm{~dB}$, see Figure $7(\mathrm{e})$, just the two structures with larger energy are identified (although not as well as in the previous cases). Note that in this case, the noise has an energy that is larger than the ones of the third and fourth structures.

\subsection{Denoising by synthesis}

As we have seen, our decomposition algorithm can reasonably identify/obtain the signal components even subject to high-level noise. Therefore, we can use the decomposition algorithm to remove the noise that may be present in the signal. To access the capability of this analysis-synthesis denoising strategy, we first generate a set of corrupted signal versions with different values of $\mathrm{SNR}_{C}$, see (11). Then, we decompose each corrupted signal version and compute the reconstruction signal-to-noise ratio

$$
\mathrm{SNR}_{\mathrm{R}}=10 \log _{10}\left(\frac{\|\mathbf{x}\|^{2}}{\|\mathbf{x}-\hat{\mathbf{x}}\|^{2}}\right)(\mathrm{dB})
$$

where $\hat{\mathbf{x}}$ is the synthesized signal (see (2)) for the different corrupted versions of $\mathbf{x}$.

Figure 8 shows $\mathrm{SNR}_{\mathrm{R}}$ in function of $\mathrm{SNR}_{\mathrm{C}}$ for the signal in the first row of Figure 6 taken from [77]. One can note that $\mathrm{SNR}_{R}$ is always larger than $\mathrm{SNR}_{\mathrm{C}}$, specially at low $\mathrm{SNR}_{\mathrm{C}}$, showing that the analysis-synthesis denoising approach is effective for signal denoising. 

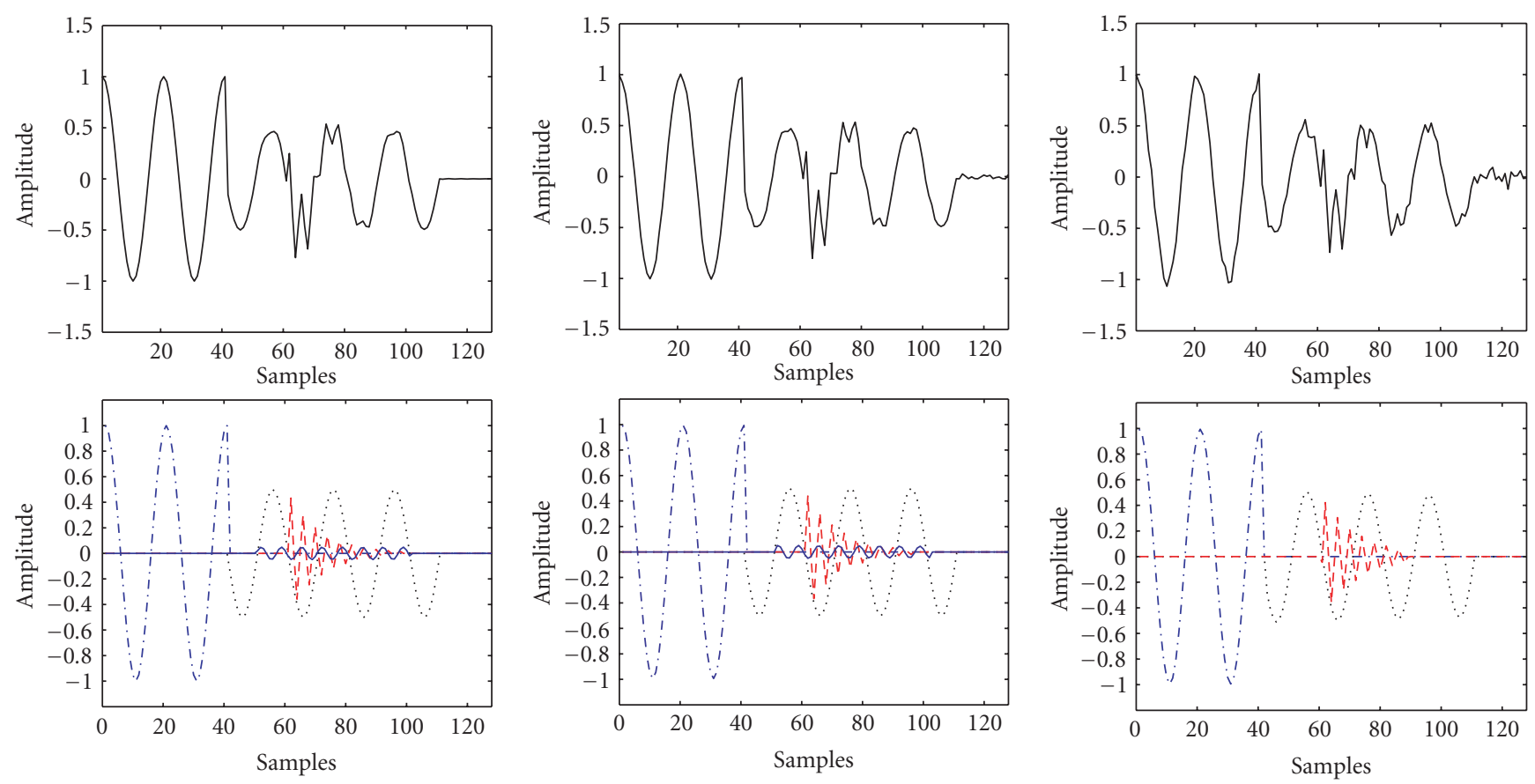

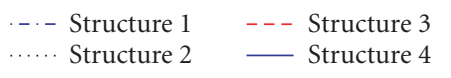

(a) Original
...- Structure 1 - - - Structure 3

..... Structure $2-$ Structure 4

(b) Subject to $\mathrm{SNR}_{\mathrm{C}}=30 \mathrm{~dB}$
- - - Structure 1

..... Structure 2

- - - Structure 3

(c) Subject to $\mathrm{SNR}_{\mathrm{C}}=20 \mathrm{~dB}$
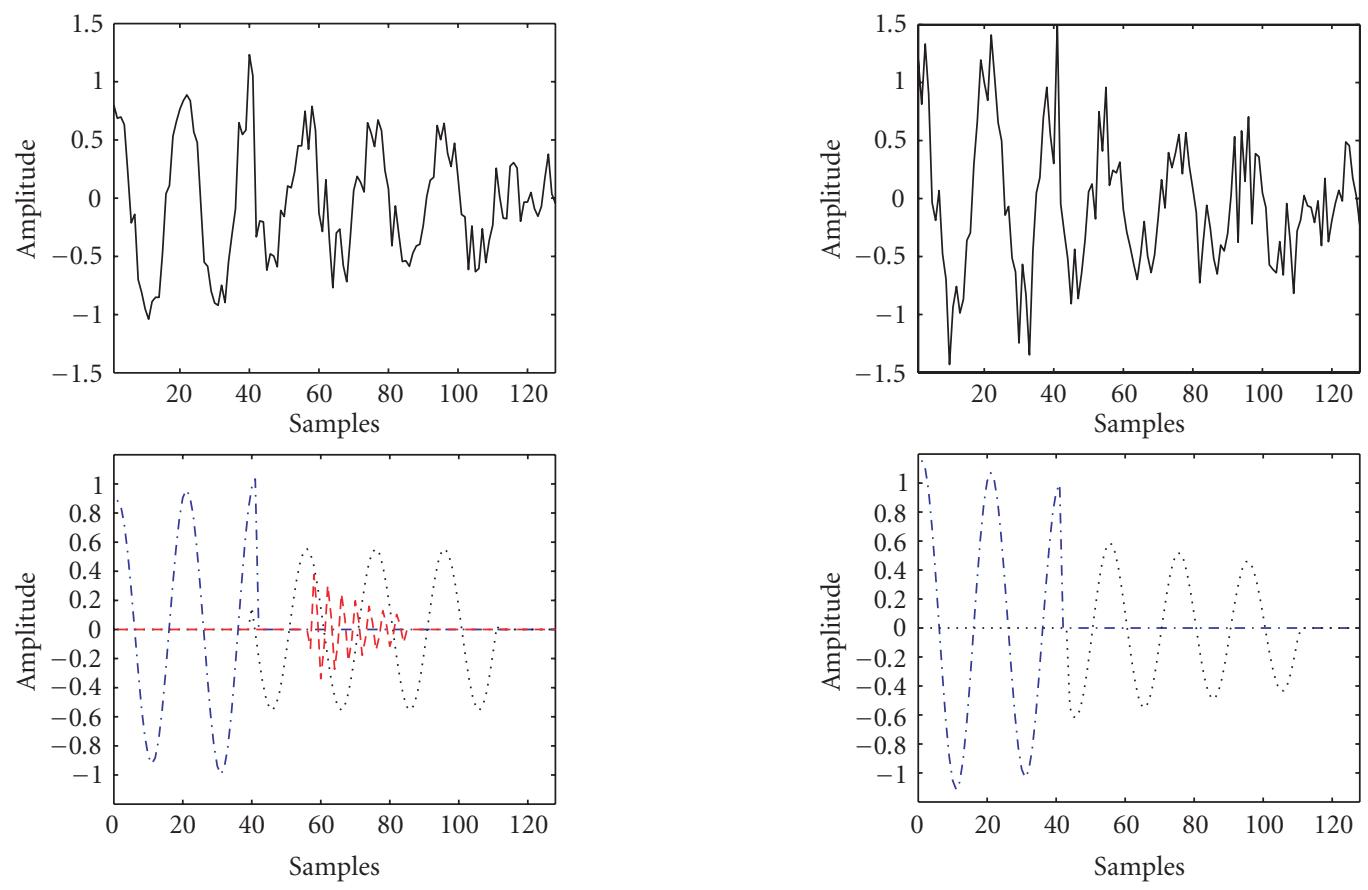

-. - Structure 1

..... Structure 2

-. - Structure 1

Structure 2

- - Structure 3

(d) Subject to $\mathrm{SNR}_{\mathrm{C}}=10 \mathrm{~dB}$

(e) Subject to $\mathrm{SNR}_{\mathrm{C}}=5 \mathrm{~dB}$

FIGURE 7: Generation of coherent signal model subject to several signal-to-noise ratios. 


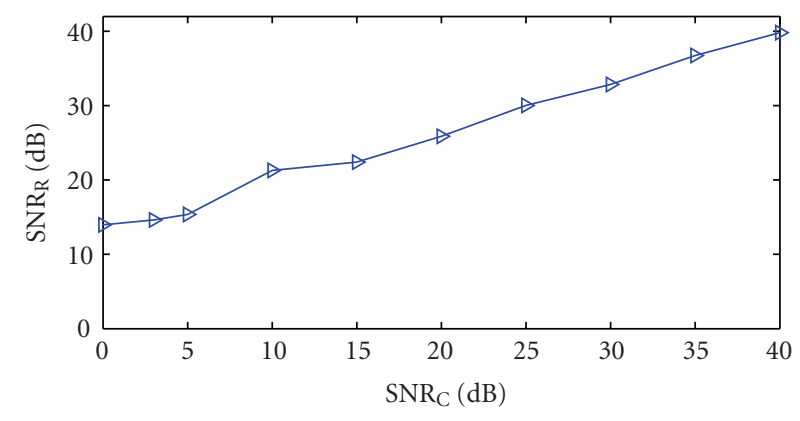

FIGURE 8: Performance of the analysis-synthesis denoising.

\subsection{Fundamental extraction and transient separation}

Several works have proposed analysis methods that start by extracting the signal's fundamental and then use the remaining signal (the transient, error or innovation signal) for analyzing the disturbance and classifying it [20, 23, 78]. Since our decomposition method automatically extracts the signal fundamental when it has a strong presence in the signal, we can subtract the fundamental from the signal in order to obtain the transient signal.

Figures 9(a) and 9(b) show examples of the above "transient separation." For example, in Figure 9(b), one can observe that our method detects the presence of a "DC" component with a transient (power event) occurring at $0.015 \mathrm{sec}$ ond.

\subsection{Filtering the "DC component"}

We now study the capability of the MP for filtering the "DC component" that sometimes appears in current quantities after the disturbance occurs [9]. A signal corrupted by a "DC component" (exponential decay) can be modeled as

$$
A e^{-\lambda t}\left[u\left(t-t^{s}\right)-u\left(t-t^{e}\right)\right]+B \sin (2 \pi F t+\phi),
$$

where $t^{s}$ and $t^{e}$ are the start and end times of the "DC component" phenomenon (for simplicity, the start and end times of the sinusoidal component are not presented) and $\lambda$ expresses the exponential decay constant. Since (13) is a particular case of (1), the decomposition algorithm presented is capable of extracting/identifying the "DC component." Once the signal is decomposed, the "DC component" can be filtered out at the signal synthesis. This filtering is achieved by ignoring in the signal synthesis all the low-pass structures (the ones with zero frequency) and that are not of impulsive nature (time support not smaller than $10 \%$ of the fundamental frequency period) obtained by in the signal analysis.

Several analyses of disturbance signals are based on comparisons of the values of current and voltage quantities, often in phasor form. For that, the signal is filtered to obtain just the fundamental frequency contribution using, for example, Fourier filters $[9,10]$. Therefore, this measurement was used to evaluate the ability of our method to filter the "DC component." An example of the "DC component" filtering on a

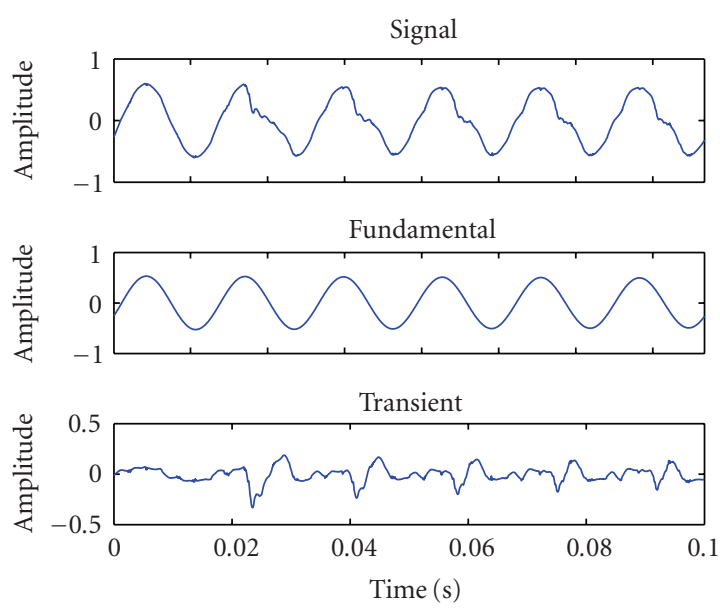

(a) Disturbance signal taken from [77]

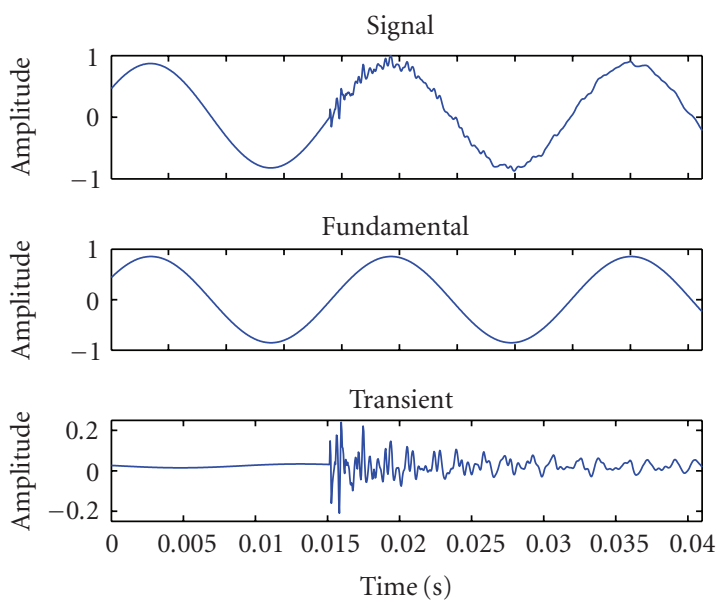

(b) Disturbance signal taken from [22]

FIGURE 9: Fundamental extraction and transient separation for disturbance signals.

synthetic signal that was generated using the model equation (1) can be seen in Figure 10. The components of the original signal are two sinusoids of $60 \mathrm{~Hz}$ with amplitudes 1 and 2 and phases $0^{\circ}$ and $90^{\circ}$ that go from samples 0 to 50 , and 50 to 100 , respectively. To the signal formed by the sum of these components, a "DC component" is added starting at sample 50 and ending at sample 100. Its decay is 0.05 and its amplitude is 3 . In Figure 10, one can see that in the filtered signal the, "DC component" is almost totally eliminated. In addition, the voltage and current phasors in the filtered signal are very close to the ones of the nondisturbed signal. This filtering has shown to be effective when applied to synthetic and natural signals as well as signals obtained through ATPEMTP [79]. Another example of this filtering process can be seen in [22]. 

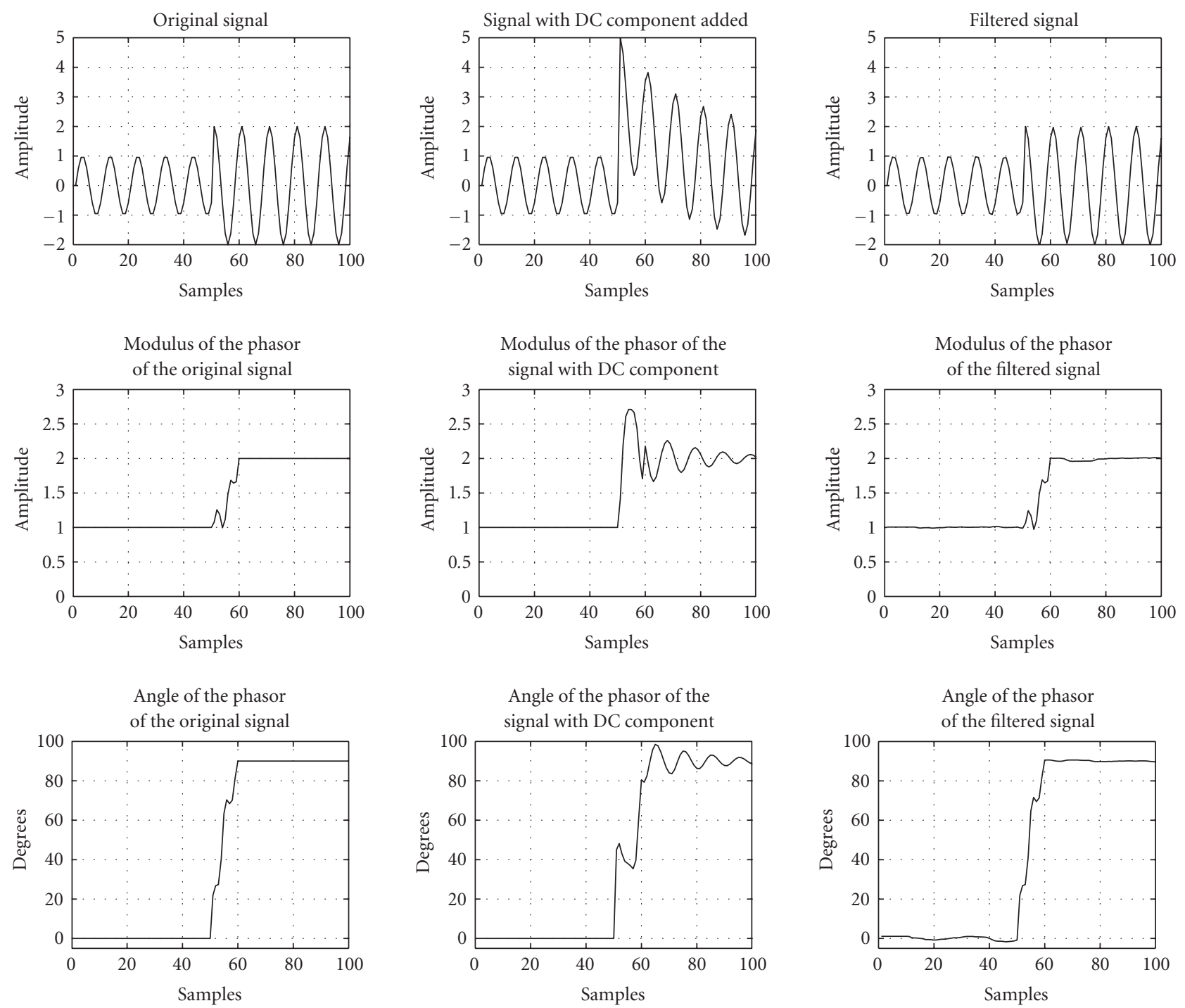

FIGURE 10: Fourier filter applied after "DC component" filtering of a synthetic signal.

\subsection{Compression of disturbance signals}

For compression, the coefficients and atom parameters need to be quantized after the decomposition process. The quantizations of the parameters and of the coefficients give rise to the reconstructed signal

$$
\tilde{\mathbf{x}}=\sum_{m=0}^{M-1} Q_{\alpha}\left\{\alpha_{m}\right\} \mathbf{g}_{Q_{i}\{\gamma(m)\}},
$$

where $Q_{\alpha}\{\cdot\}$ is the quantizer of the coefficients and $Q_{i}\{\cdot\}$ denotes the quantizer of the parameters. Each different quantization rule $Q_{i}\{\cdot\}$ corresponds to a distinct dictionary $D_{i} \subset D$ ( $D$ is the original continuous parameter dictionary). That is, the dictionary $D_{i}$ is defined by the mapping $Q_{i}\{\cdot\}$ and $\tilde{\mathbf{x}}$ corresponds to a weighted sum of its elements. The weights of the atoms in $\tilde{\mathbf{x}}$ depend on the quantizer of the coefficients
$Q_{\alpha}\{\cdot\}$. Figure 11 illustrates this compression framework. The optimum rate $\times$ distortion solution for this compression scheme is provided by finding the quantizers $Q_{\alpha}\{\cdot\}$ and $Q_{i}\{\cdot\}$ that lead to the minimum distortion for a given rate.

Signal compression based on the MP usually retains a certain number of terms $M$ and quantizes just the coefficients [71]. The compression framework we employ substantially differs from these. Since we use a dictionary of some continuous parameters, for compression it is necessary to quantize the parameters of atoms. This is equivalent to using multiple dictionaries in the decomposition process and selecting one of them for coding a given signal.

Rate $\times$ distortion optimization is employed in compression systems to achieve the best signal reproduction for a desired compression target [80]. In the framework at hand, one has to find a compromise between the number of atoms in the signal representation, the quantization of the coefficients, 


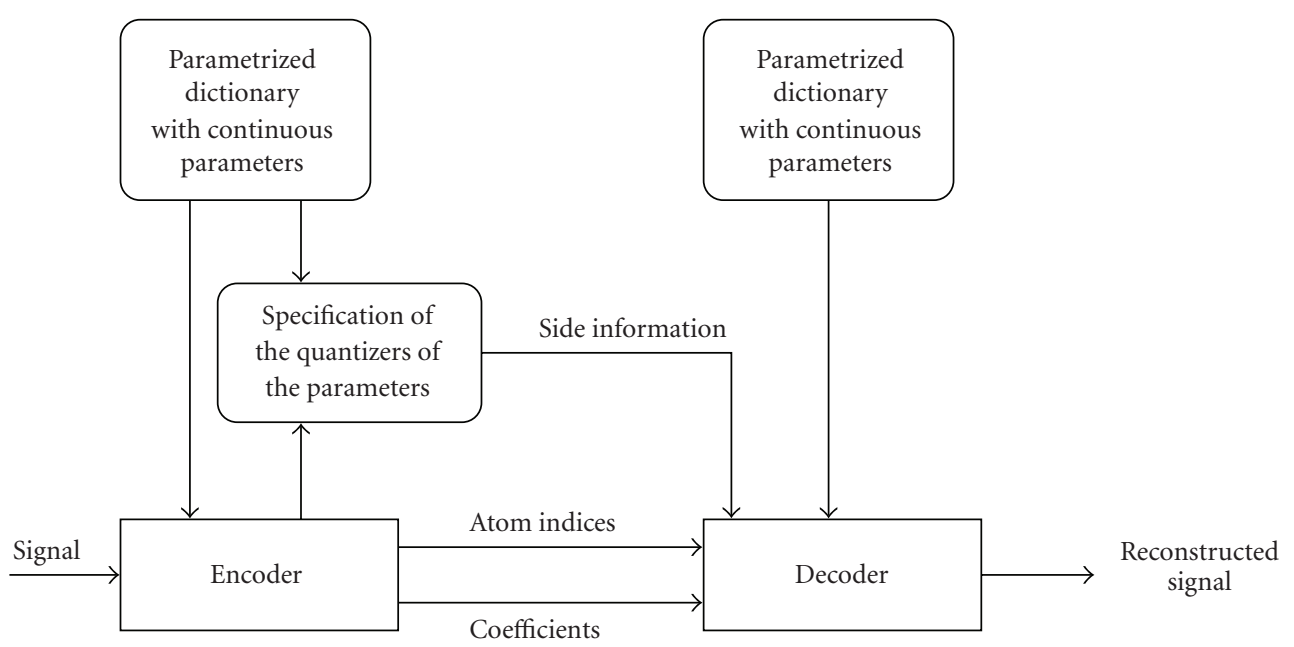

FIGURE 11: Compression framework by quantizing the parameters of the atoms.

and the choice of the dictionary $D_{i} \in D$ that is defined by the quantizers of the atom parameters.

Define the number of bits for a given atom as

$$
r=r_{\alpha}+r_{\xi}+r_{\rho}+r_{\phi}+r_{n^{s}}+r_{n^{e}}
$$

where $r_{\alpha}, r_{\xi}, r_{\rho}, r_{\phi}, r_{n^{s}}$, and $r_{n^{e}}$ denote the amount of bits allocated to $\alpha, \xi, \rho, \phi, n^{s}$, and $n^{e}$, respectively. The total number of bits spent for coding a signal, except for side information, will be $r \times M$, where $M$ is the number of terms in the signal reconstruction (see (14)). The starting and ending samples of the atoms $n_{m}^{s}$ and $n_{m}^{e}$ are coded using $\log _{2}(N)$ bits as the decomposition algorithm limits them to integers in this range, meaning that $r_{n^{s}}=r_{n^{e}}=\log _{2}(N)$ ( $N$ is the signal length). In addition, since the decomposition algorithm already delivers a quantized frequency $\xi_{m}$ using multiples of the fundamental one, the frequency of the atoms are coded using $r_{\xi}=\log _{2}\left(\left(F_{s} / 2\right) / F\right)$ bits. Therefore, the total distortion is expressed just as a function of the number of bits spent on the coefficients, the decaying factors, and the phases of the atoms, resulting in

$$
d=f\left(r_{\alpha}, r_{\rho}, r_{\phi}\right), \quad \operatorname{MSE}=\frac{1}{N}\|\mathbf{x}-\tilde{\mathbf{x}}\|^{2},
$$

where MSE means mean-squared error.

In [81], an efficient rate-distortion optimization strategy based on a training stage is presented for the decompositions obtained here. This optimization finds the number of levels of uniform quantizers (restricting the number of levels of the quantizers to powers of two) for $\rho, \phi$, and $\alpha$ that jointly lead to the best rate $\times$ distortion compromise. The distortion considered in this approach is the one defined in (16) subject to the rate (in bits/sample)

$$
R_{s}=\frac{M \times r+\text { bits }_{\text {info }}}{N},
$$

where $M$ is the number of coded atoms, $r$ is given by (15), bits $_{\text {info }}$ is the number of bits spent to send side information on the quantizers design, and $N$ is the signal length.

Figure 12 shows two examples of compressed signals for two different signals taken from [77]. The signal in Figure $12(\mathrm{a})$ is compressed using a rate of 0.492188 bits/ sample with $\mathrm{SNR}_{\mathrm{R}}=24.888 \mathrm{~dB}$ and the signal in Figure 12(b) is compressed using 0.542969 bits/sample with $\mathrm{SNR}_{\mathrm{R}}=25.387 \mathrm{~dB}$. The rate $\times$ distortion performance of the compression method for the signal in Figure 12(a) is shown in Figure 13(a), while for the signal in Figure 12(b) it is shown in Figure 13(b). As one can see from these graphs, the compression method described obtains high reproduction quality at low bit rates.

\section{CONCLUSION}

This paper reviewed an atomic decomposition algorithm that decomposes disturbance signals by means of a linear combination of damped sinusoids having frequencies in multiples of the system fundamental frequency.

The decomposition algorithm discussed obtains a coherent decomposition of the signal. Therefore, it can be applied for signal denoising, extraction of the fundamental frequency, and separation of the transient signal. The proposed signal decomposition can also be used to filter out the "DC component" that often impairs the location of the fault in the transmission line when phasorial techniques are employed for that purpose. In addition, the decompositions can be used for signal compression at low bit rates and high signalto-noise ratio, thus keeping the relevant information in the compressed version of the disturbance signal.

Under current investigation, we show how to use the disturbance signal modeling by means of damped sinusoid as input in expert systems for automatic classification of 


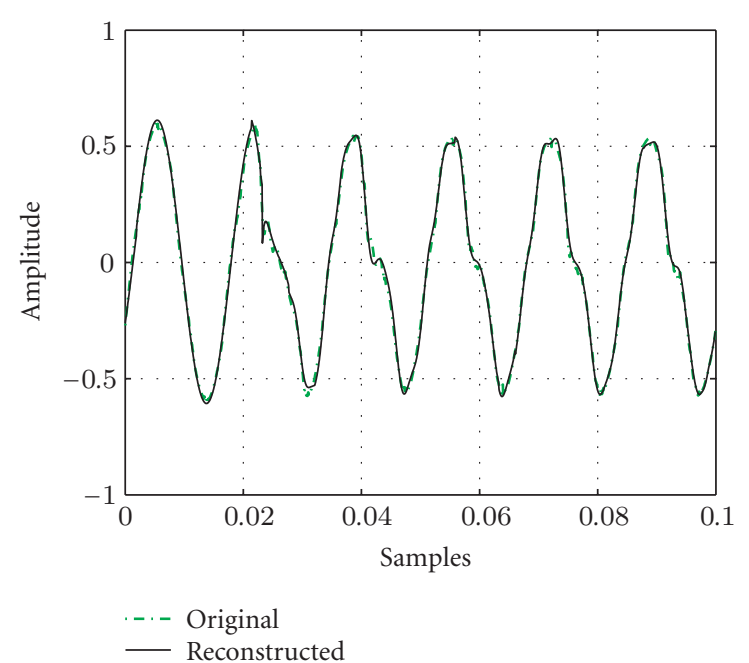

(a)

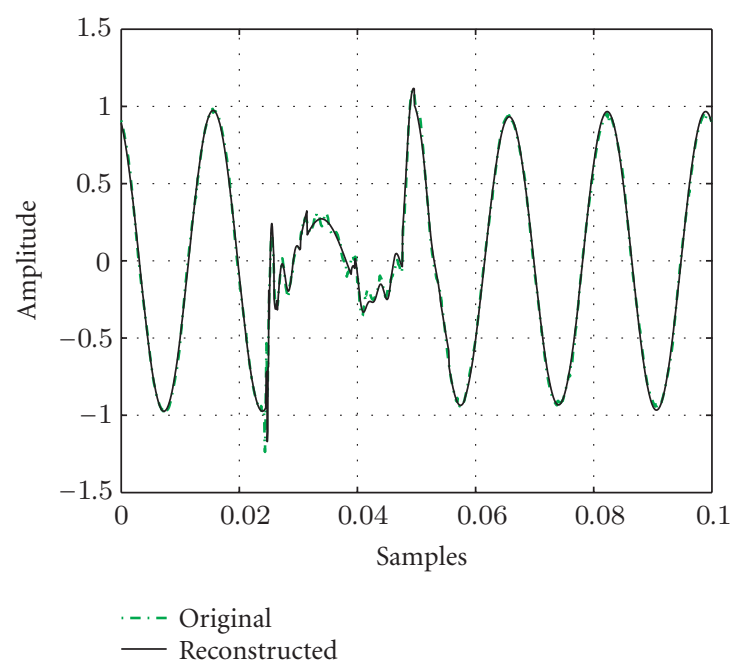

(b)

FIgURE 12: Examples of the compression performance for two signals from [77].

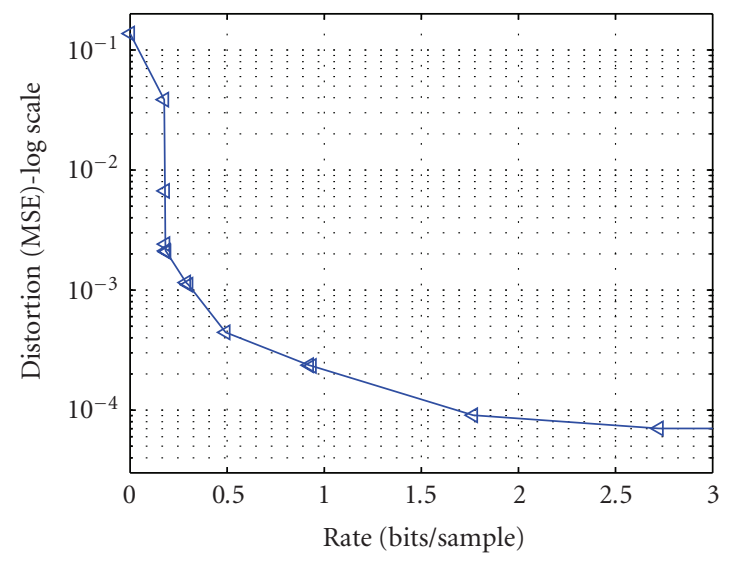

(a)

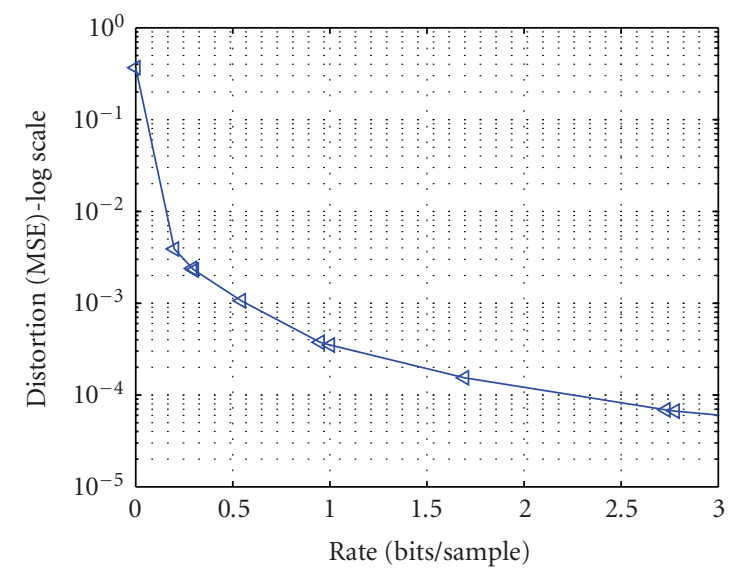

(b)

FIGURE 13: Rate $\times$ distortion performance of the compression system presented for two signals from [77].

faults and disturbances. In addition, we are developing a methodology to evaluate compression systems for disturbance signals using the techniques normally employed for analyzing these signals.

\section{REFERENCES}

[1] M. A. M. Rodrigues, M. V. F. de Figueiredo, A. L. L. Miranda, and S. S. Diniz, "Oscillography for power system operational planning," in Proceedings of the 7th Symposium of Specialists in Electric Operational and Expansion Planning (VII SEPOPE '00), Curitiba, Brazil, May 2000.

[2] J. Arrillaga, M. H. J. Bollen, and N. R. Watson, "Power quality following deregulation," Proceedings of the IEEE, vol. 88, no. 2, pp. 246-261, 2000.
[3] R. C. Dugan, M. F. McGranghan, and H. W. Beaty, Electrial Power Systems Quality, McGraw-Hill, New York, NY, USA, 1996.

[4] M. H. J. Bollen, Understanding Power Quality ProblemsVoltage Sags and Interruptions, IEEE Press, Piscataway, NJ, USA, 2000.

[5] N. Ruiz-Reyes, P. Vera-Candeas, and F. Jurado, "Discrimination between transient voltage stability and voltage sag using damped sinusoids-based transient modeling," IEEE Transactions on Power Delivery, vol. 20, no. 4, pp. 2644-2650, 2005.

[6] M. Kezunovic and I. Rikalo, "Automating the analysis of faults and power quality," IEEE Computer Applications in Power, vol. 12 , no. 1, pp. 46-50, 1999.

[7] B. J. Bujanowski, J. W. Pierre, S. M. Hietpas, T. L. Sharpe, and D. A. Pierre, "A comparison of several system identification methods with application to power systems," in Proceedings of 
the 36th Midwest Symposium on Circuits and Systems (MWSCAS '93), vol. 1, pp. 64-67, Detroit, Mich, USA, August 1993.

[8] W. R. A. Ibrahim and M. M. Morcos, "Artificial intelligence and advanced mathematical tools for power quality applications: a survey," IEEE Transactions on Power Delivery, vol. 17, no. 2, pp. 668-673, 2002.

[9] E. O. Schweitzer III and D. Hou, "Filtering for protective relays," in Proceedings of the 47th Annual Georgia Tech Protective Relaying Conference, Atlanta, Ga, USA, April 1993.

[10] D. Wiot, "A new adaptive transient monitoring scheme for detection of power system events," IEEE Transactions on Power Delivery, vol. 19, no. 1, pp. 42-48, 2004.

[11] T. Lobos, J. Rezmer, and H.-J. Koglin, "Analysis of power system transients using wavelets and Prony method," in Proceedings of IEEE Porto Power Tech Conference (PTC '01), vol. 4, p. 4, Porto, Portugal, September 2001.

[12] M. M. Tawfik and M. M. Morcos, "ANN-based techniques for estimating fault location on transmission lines using Prony method," IEEE Transactions on Power Delivery, vol. 16, no. 2, pp. 219-224, 2001.

[13] A. W. Galli, G. T. Heydt, and P. F. Ribeiro, "Exploring the power of wavelet analysis," IEEE Computer Applications in Power, vol. 9, no. 4, pp. 37-41, 1996.

[14] J. Chung, E. J. Powers, W. M. Grady, and S. C. Bhatt, "Electric power transient disturbance classification using waveletbased hidden Markov models," in Proceedings of IEEE International Conference on Acoustics, Speech and Signal Processing (ICASSP '00), vol. 6, pp. 3662-3665, Istanbul, Turkey, June 2000.

[15] P. Pillaya and A. Bhattacharjee, "Application of wavelets to model short-term power system disturbances," IEEE Transactions on Power Systems, vol. 11, no. 4, pp. 2031-2037, 1996.

[16] O. Poisson, P. Rioual, and M. Meunier, "Detection and measurement of power quality disturbances using wavelet transform," IEEE Transactions on Power Delivery, vol. 15, no. 3, pp. 1039-1044, 2000.

[17] H.-T. Yang and C.-C. Liao, "A de-noising scheme for enhancing wavelet-based power quality monitoring system," IEEE Transactions on Power Delivery, vol. 16, no. 3, pp. 353-360, 2001.

[18] S. Santoso, W. M. Grady, E. J. Powers, J. Lamoree, and S. C. Bhatt, "Characterization of distribution power quality events with Fourier and wavelet transforms," IEEE Transactions on Power Delivery, vol. 15, no. 1, pp. 247-254, 2000.

[19] M. Karimi, H. Mokhtari, and M. R. Iravani, "Wavelet based on-line disturbance detection for power quality applications," IEEE Transactions on Power Delivery, vol. 15, no. 4, pp. 1212$1220,2000$.

[20] C. A. Duque, M. V. Ribeiro, F. R. Ramos, and J. Szczupak, "Power quality event detection based on the divide and conquer principle and innovation concept," IEEE Transactions on Power Delivery, vol. 20, no. 4, pp. 2361-2369, 2005.

[21] M. V. Ribeiro, J. M. T. Romano, and C. A. Duque, "An improved method for signal processing and compression in power quality evaluation," IEEE Transactions on Power Delivery, vol. 19, no. 2, pp. 464-471, 2004.

[22] L. Lovisolo, E. A. B. da Silva, M. A. M. Rodrigues, and P. S. R. Diniz, "Efficient coherent adaptive representations of monitored electric signals in power systems using damped sinusoids," IEEE Transactions on Signal Processing, vol. 53, no. 10 I, pp. 3831-3846, 2005.

[23] M. Karimi-Ghartemani and M. R. Iravani, "A signal processing module for power system applications," IEEE Transactions on Power Delivery, vol. 18, no. 4, pp. 1118-1126, 2003.
[24] S. Mallat and Z. Zhang, "Matching pursuits with timefrequency dictionaries," IEEE Transactions on Signal Processing, vol. 41, no. 12, pp. 3397-3415, 1993.

[25] S. Mallat, A Wavelet Tour of Signal Processing, Academic Press, San Diego, Calif, USA, 1st edition, 1998.

[26] S. E. Ferrando, L. A. Kolasa, and N. Kovačević, "Algorithm 820: a flexible implementation of matching pursuit for Gabor functions on the interval," ACM Transactions on Mathematical Software, vol. 28, no. 3, pp. 337-353, 2002.

[27] M. M. Goodwin and M. Vetterli, "Matching pursuit and atomic signal models based on recursive filter banks," IEEE Transactions on Signal Processing, vol. 47, no. 7, pp. 1890-1902, 1999.

[28] R. Gribonval and E. Bacry, "Harmonic decomposition of audio signals with matching pursuit," IEEE Transactions on Signal Processing, vol. 51, no. 1, pp. 101-111, 2003.

[29] W. Xu, "Component modeling issues for power quality assessment," IEEE Power Engineering Review, vol. 21, no. 11, pp. 1215, 17, 2001.

[30] J. J. Collins and W. G. Hurley, "Application of expert systems and neural networks to the diagnosis of power quality problems," in Proceedings of the EPRI-PQA Conference, Amsterdam, The Netherlands, October 1994.

[31] A. K. Ghosh and D. L. Lubkeman, "The classification of power system disturbance waveforms using a neural network approach," IEEE Transactions on Power Delivery, vol. 10, no. 1, pp. 109-115, 1995.

[32] L. Lovisolo, E. A. B. da Silva, M. A. M. Rodrigues, and P. S. R. Diniz, "Coherent decompositions of power systems signals using damped sinusoids with applications to denoising," in Proceedings of IEEE International Symposium on Circuits and Systems (ISCAS '02), vol. 5, pp. 685-688, Phoenix, Ariz, USA, May 2002.

[33] M. A. M. Rodrigues, Efficient decompositions for signal coding, Ph.D. thesis, COPPE/UFRJ, Rio de Janeiro, RJ, USA, March 1999.

[34] B. Friedlander and B. Porat, "Detection of transient signals by the Gabor representation," IEEE Transactions on Acoustics, Speech, and Signal Processing, vol. 37, no. 2, pp. 169-180, 1989.

[35] B. Friedlander and A. Zeira, "Oversampled Gabor representation for transient signals," IEEE Transactions on Signal Processing, vol. 43, no. 9, pp. 2088-2094, 1995.

[36] M. Zibulski and Y. Y. Zeevi, "Discrete multiwindow Gabortype transforms," IEEE Transactions on Signal Processing, vol. 45, no. 6, pp. 1428-1442, 1997.

[37] I. Kauppinen, J. Kauppinen, and P. Saarinen, "A method for long extrapolation of audio signals," Journal of the Audio Engineering Society, vol. 49, no. 12, pp. 1167-1180, 2001.

[38] Y. Lu, S. Joshi, and J. M. Morris, "Noise reduction for NMR FID signals via Gabor expansion," IEEE Transactions on Biomedical Engineering, vol. 44, no. 6, pp. 512-528, 1997.

[39] S. M. Kay, Modern Spectral Estimation, Prentice-Hall, Englewood Cliffs, NJ, USA, 1988.

[40] D. W. Tufts and R. Kumaresan, "Improved spectral resolution," Proceedings of the IEEE, vol. 68, no. 3, pp. 419-421, 1980.

[41] T. J. Abatzoglou, "A fast maximum likelihood algorithm for frequency estimation of a sinusoid based on Newton's method," IEEE Transactions on Acoustics, Speech, and Signal Processing, vol. 33, no. 1, pp. 77-89, 1985.

[42] R. Kumaresan and D. W. Tufts, "Estimating the parameters of exponentially damped sinusoids and pole-zero modeling in noise," IEEE Transactions on Acoustics, Speech, and Signal Processing, vol. 30, no. 6, pp. 833-840, 1982. 
[43] Y. Hua and T. K. Sarkar, "Matrix pencil method for estimating parameters of exponentially damped/undamped sinusoids in noise," IEEE Transactions on Acoustics, Speech, and Signal Processing, vol. 38, no. 5, pp. 814-824, 1990.

[44] C. K. Papadopoulos and C. L. Nikias, "Parameter estimation of exponentially damped sinusoids using higher order statistics," IEEE Transactions on Acoustics, Speech, and Signal Processing, vol. 38, no. 8, pp. 1424-1436, 1990.

[45] Y. Hua, "Parameter estimation of exponentially damped sinusoids using higher order statistics and matrix pencil," IEEE Transactions on Signal Processing, vol. 39, no. 7, pp. 1691-1692, 1991.

[46] D. P. Ruiz, M. C. Carrion, A. Gallego, and A. Medouri, "Parameter estimation of exponentially damped sinusoids using a higher order correlation-based approach," IEEE Transactions on Signal Processing, vol. 43, no. 11, pp. 2665-2677, 1995.

[47] M. V. Ribeiro, S. H. Park, J. M. T. Romano, and S. K. Mitra, "A novel MDL-based compression method for power quality applications," IEEE Transactions on Power Delivery, vol. 22, no. 1, pp. 27-36, 2007.

[48] H. Krim, D. Tucker, S. Mallat, and D. Donoho, "On denoising and best signal representation," IEEE Transactions on Information Theory, vol. 45, no. 7, pp. 2225-2238, 1999.

[49] P. Vera-Candeas, N. Ruiz-Reyes, M. Rosa-Zurera, D. MartinezMunoz, and F. Lopez-Ferreras, "Transient modeling by matching pursuits with a wavelet dictionary for parametric audio coding," IEEE Signal Processing Letters, vol. 11, no. 3, pp. 349$352,2004$.

[50] R. Heusdens, R. Vafin, and W. B. Kleijn, "Sinusoidal modeling using psychoacoustic-adaptive matching pursuits," IEEE Signal Processing Letters, vol. 9, no. 8, pp. 262-265, 2002.

[51] S. Jaggi, W. C. Karl, S. Mallat, and A. S. Willsky, "High resolution pursuit for feature extraction," Applied and Computational Harmonic Analysis, vol. 5, no. 4, pp. 428-449, 1998.

[52] M. M. Goodwin, Adaptive Signal Models: Theory, Algorithms, and Audio Applications, Kluwer International Series in Engineering and Computer Science, Kluwer Academic, New York, NY, USA, 1st edition, 1998.

[53] D. L. Donoho, M. Vetterli, R. A. DeVore, and I. Daubechies, "Data compression and harmonic analysis," IEEE Transactions on Information Theory, vol. 44, no. 6, pp. 2435-2476, 1998.

[54] K. Engan, S. O. Aase, and J. H. Husøy, "Multi-frame compression: theory and design," Signal Processing, vol. 80, no. 10, pp. 2121-2140, 2000.

[55] O. K. Al-Shaykh, E. Miloslavsky, T. Nomura, R. Neff, and A. Zakhor, "Video compression using matching pursuits," IEEE Transactions on Circuits and Systems for Video Technology, vol. 9, no. 1, pp. 123-143, 1999.

[56] R. Neff and A. Zakhor, "Modulus quantization for matchingpursuit video coding," IEEE Transactions on Circuits and Systems for Video Technology, vol. 10, no. 6, pp. 895-912, 2000.

[57] R. Caetano, E. A. B. da Silva, and A. G. Ciancio, "Matching pursuits video coding using generalized bit-planes," in Proceedings of International Conference on Image Processing (ICIP'02), vol. 3, pp. 677-680, Rochester, NY, USA, September 2002.

[58] R. Gribonval, "From projection pursuit and CART to adaptive discriminant analysis?" IEEE Transactions on Neural Networks, vol. 16, no. 3, pp. 522-532, 2005.

[59] V. K. Goyal, M. Vetterli, and N. T. Thao, "Quantized overcomplete expansions in $\mathbb{R}^{N}$ : analysis, synthesis, and algorithms," IEEE Transactions on Information Theory, vol. 44, no. 1, pp. 16-31, 1998.
[60] G. Davis, Adaptive nonlinear approximations, Ph.D. thesis, New York University, New York, NY, USA, 1994.

[61] J. A. Tropp, "Greed is good: algorithmic results for sparse approximation," IEEE Transactions on Information Theory, vol. 50, no. 10, pp. 2231-2242, 2004.

[62] J. Adler, B. D. Rao, and K. Kreutz-Delgado, "Comparison of basis selection methods," in Proceedings of the 30th Asilomar Conference on Signals Conference on Signals, Systems \& Computers (ACSSC '96), vol. 1, pp. 252-257, Pacific Grove, Calif, USA, November 1997.

[63] Z. Zhang, "Matching pursuits," Ph.D. dissertation, New York University, New York, NY, USA, 1993.

[64] J. B. Kruskal, "Toward a practical method to hel uncover the structure of a set of multivariate observations by finding the linear transformation which optimizes a new "index of condensation"'” in Statistical Computation, R. C. Milton and J. A. Nelder, Eds., Academic Press, New York, NY, USA, 1969.

[65] J. H. Friedman and W. Suetzle, "Projection pursuit regression," Journal of the American Statiscal Association, vol. 76, no. 376, pp. 817-823, 1981.

[66] S. Chen, S. A. Billings, and W. Luo, "Orthogonal least squares methods and their application to non-linear system identification," International Journal of Control, vol. 50, no. 5, pp. 18731896, 1989.

[67] R. A. DeVore, "Nonlinear approximation," Acta Numerica, vol. 7, pp. 51-150, 1998.

[68] V. N. Temlyakov, "Nonlinear methods of approximation," Foundations of Computational Mathematics, vol. 3, no. 1, pp. 33-107, 2003.

[69] R. A. DeVore and V. N. Temlyakov, "Some remarks on greedy algorithms," Advances in Computational Mathematics, vol. 5, no. 1, pp. 173-187, 1996.

[70] R. Gribonval, E. Bacry, S. Mallat, P. Depalle, and X. Rodet, "Analysis of sound signals with high resolution matching pursuit," in Proceedings of the IEEE-SP International Symposium on Time-Frequency and Time-Scale Analysis (TFTSA '96), pp. 125-128, Paris, France, June 1996.

[71] P. Frossard, P. Vandergheynst, R. M. Figueras I Ventura, and M. Kunt, "A posteriori quantization of progressive matching pursuit streams," IEEE Transactions on Signal Processing, vol. 52, no. 2, pp. 525-535, 2004.

[72] P. J. Durka, D. Ircha, and K. J. Blinowska, "Stochastic timefrequency dictionaries for matching pursuit," IEEE Transactions on Signal Processing, vol. 49, no. 3, pp. 507-510, 2001.

[73] A. Papandreou-Suppappola and S. B. Suppappola, "Analysis and classification of time-varying signals with multiple timefrequency structures," IEEE Signal Processing Letters, vol. 9, no. 3, pp. 92-95, 2002.

[74] S. E. Ferrando, E. J. Doolittle, A. J. Bernal, and L. J. Bernal, "Probabilistic matching pursuit with Gabor dictionaries," Signal Processing, vol. 80, no. 10, pp. 2099-2120, 2000.

[75] I. Daubechies, Ten Lectures on Wavelets, SIAM, Philadelphia, Pa, USA, 1991.

[76] C. O. Etemoǧlu and V. Cuperman, "Matching pursuits sinusoidal speech coding," IEEE Transactions on Speech and Audio Processing, vol. 11, no. 5, pp. 413-424, 2003.

[77] "IEEE PES Working Group 1433 Power Quality," http:// grouper.ieee.org/groups/1433/.

[78] A. S. Cerqueira, C. A. Duque, R. M. Trindade, and M. V. Ribeiro, "Digital system for detection and classification of electrical events," in Proceedings of IEEE International 
Symposium on Circuits and Systems (ISCAS '05), vol. 6, pp. 5417-5420, Kobe, Japan, May 2005.

[79] Canadian-American EMTP User Group, "EMTP Rule Book, Alternative Transients Rule Book," Canadian-American EMTP User Group, 1987-1992.

[80] A. Ortega and K. Ramchandran, "Rate-distortion methods for: image and video compression," IEEE Signal Processing Magazine, vol. 15, no. 6, pp. 23-50, 1998.

[81] M. P. Tcheou, L. Lovisolo, E. A. B. da Silva, M. A. M. Rodrigues, and P. S. R. Diniz, "Optimum rate-distortion dictionary selection for compression of atomic decompositions of electric disturbance signals," IEEE Signal Processing Letters, vol. 14, no. 2, pp. 81-84, 2007.

Lisandro Lovisolo was born in Neuquen, Argentina. He received the Engineering degree in electronics in 1999 and the M.S. and D.S. degrees in electrical engineering in 2001 and 2006, respectively, all from the Federal University of Rio de Janeiro. Since 2003, he has been with the Department of Electronics and Telecommunications Engineering at University of the State of Rio de Janeiro (UERJ). His research interests are in signal processing, especially adaptive and overcomplete signal/image decompositions for analysis and coding purposes.

Michel P. Tcheou was born in Rio de Janeiro, Brazil. He received the Engineering degree in electronics, in 2003, and the M.S. degree in electrical engineering, in 2005, from Federal University of Rio de Janeiro (UFRJ), Rio de Janeiro, Brazil. He is currently pursuing the D.S. degree at the Signal Processing Laboratory (LPS), UFRJ. In 2006, he joined the Electric Power Research Center (CEPEL). His research interests are in signal processing, especially atomic decompositions with applications to multimedia and power systems.

Eduardo A. B. da Silva was born in Rio de Janeiro, Brazil, in 1963. He received the Engineering degree in electronics from Instituto Militar de Engenharia (IME), Brazil, in 1984, the M.S. degree in electrical engineering from Universidade Federal do Rio de Janeiro (COPPE/UFRJ) in 1990, and the Ph.D. degree in electronics from the University of Essex, England, in 1995. In 1987 and 1988, he was with the Department of

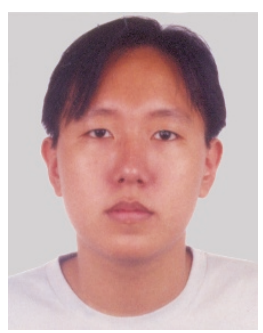
Electrical Engineering at Institute Militar de Engenharia, Rio de Janeiro, Brazil. Since 1989, he has been with the Department of Electronics Engineering, UFRJ. He has also been with the Department of Electrical Engineering, COPPE/UFRJ, since 1996. He is coauthor of the book "Digital Signal Processing-System Analysis and Design," published by Cambridge University Press in 2002. He has served as Associate Editor of the IEEE Transactions on Circuits and Systems Part I and Part II. He has been a Distinguished Lecturer of the IEEE Circuits and Systems Society in 2003 and 2004. His research interests lie in the fields of digital signal and image processing, especially signal compression, digital television, wavelet transforms, mathematical morphology, and applications to telecommunications. He is a Senior Member of the IEEE.
Marco A. M. Rodrigues was born in Rio de Janeiro, Brazil, in 1964. He received the Engineering degree in electronics, the M.S. and the D.S. degrees in electrical engineering from the University of Rio de Janeiro in 1986, 1991, and 1999, respectively. He has worked at the Electric Power Research Center (CEPEL) in Rio de Janeiro, Brazil, since 1987, in data acquisition systems design, software design, algorithms for data

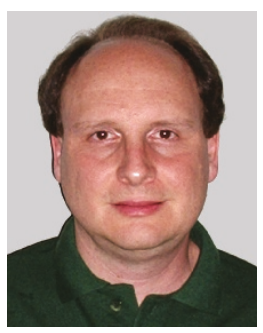
analysis, and control and signal processing applications related to power systems. He is also an Invited Professor in a postgraduate course in protection for power systems, held at the University of Rio de Janeiro. His current research interests are signal processing, power system measurements, oscillographic analysis automation, and power system protection systems. Dr. Rodrigues is Senior Member of IEEE and Member of Cigré (Conseil International des Grands Réseaux Électriques) and of SBrT (the Brazilian Telecommunications Society). He participated in the steering committee of the Brazilian Protection and Control Technical Seminary (STPC) in 2003 and 2005.

Paulo S. R. Diniz was born in Niteroi, Brazil. He received the Electronics Eng. degree (cum laude) from the Federal University of Rio de Janeiro (UFRJ) in 1978, the M.S. degree from COPPE/UFRJ in 1981, and the Ph.D. degree from Concordia University, Montreal, PQ, Canada, in 1984, all in electrical engineering. Since 1979, he has been with the Department of Electronic Engineering (the Undergraduate Depart-

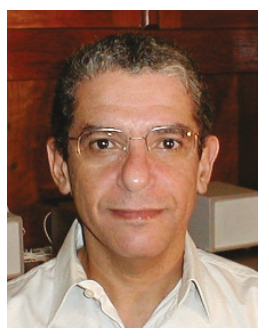
ment), UFRJ. He has also been with the Program of Electrical Engineering (the Graduate Studies Department), COPPE/UFRJ, since 1984, where he is presently a Professor. He wrote the books "Adaptive Filtering: Algorithms and Practical Implementation," Springer, Third Edition 2007, and "Digital Signal Processing: System Analysis and Design," Cambridge University Press, Cambridge, UK, 2002 (with E. A. B. da Silva and S. L. Netto). He is also a Fellow of IEEE and has served as an Associate Editor for several Journals. He served as Distinguished Lecturer of the IEEE Signal Processing Society and received the 2004 Education Award of the IEEE Circuits and Systems Society. 